\title{
LAD: A Layout Anomaly Detector for Android Applications
}

\author{
Cheng-Zen Yang, Chih-Ju Lai, Peng Lu, and Zhi-Jun You \\ Department of Computer Science and Engineering \\ Yuan Ze University \\ Chungli, Taiwan, R. O. C. \\ \{czyang,cj116,pl16,zjy19\}@syslab.cse.yzu.edu.tw
}

\begin{abstract}
In recent years, Android has become one of the most popular mobile operating systems. Software testing of Android applications becomes a very important issue. In the past research, many studies focus on functional testing. Recently, some studies start to discuss the layout anomaly issue. However, these schemes investigate few types of layout anomalies. Moreover, there may exist underlying platform constraints. In this paper, a detection tool LAD (Layout Anomaly Detector) is proposed. LAD considers the anomaly types studied in the previous work with a newly proposed anomaly type for scale maladaptation of components and text. LAD also supports testing scripts. We have conducted empirical experiments with four real apps. The experimental results show that LAD can effectively detect the layout anomalies of these apps.
\end{abstract}

Keywords-Android; layout anomaly; GUI layout testing; automatic testing

\section{INTRODUCTION}

As shown in the statistics of AppBrain, the number of Android apps has exceeded 2.59 million on Google Play in March 2019 [1]. In the past, automatic Android software testing has received many attentions, such as [2-5]. However, most of the previous studies focus on functional testing. To our best knowledge, the number of research studies on detecting layout anomalies for Android apps is comparatively few [6-9]. Because Android devices have the severe fragmentation problem, layout anomalies become an important issue for user experience.

The layout anomaly problem occurs in an app if its layout design does not consider the diversity of different screen specifications. This problem can be very annoying for users and app developers. For example, the layout anomalies such as UI component overlap or cropped text may hamper the user operations. As reported in [8], some UI components may even disappear when the app is executed in a smaller screen size.

To tackle layout anomalies, app developers need to take a lot of time to inspect the layouts for many screen specifications. As illustrated in $[8,9]$, if there are $m$ layouts in an app and $n$ screen specifications, the time complexity of the inspection work is $O(m \times n)$. This workload is harmless if $n$ is very small. However, a survey of OpenSignal shows that over 150 different screen sizes have existed for Android devices in 2014 [10]. If there are 100 different screen specifications to be inspected and the inspection time is 5 minutes per layout, the total inspection time will be 83 hours for an app of 10 layouts.
In recent years, studies have been conducted to deal with the problem of layout anomaly detection. Several tools like Galen Framework [6] and ITArray Automotion Framework [7] are developed to perform visual testing for Android apps. Their users need either to write scripts to specify the geometry relationships or to import the customized Java code into the app under testing (AUT). In 2017, Shih et al. proposed an automatic detection tool called UI-Explorer to detect two types of layout anomalies [8]. In UI-explorer, all layouts are traversed and inspected automatically. However, UI-explorer provides only click events to traverse the layouts. It lacks an approach to handle user inputs. Hasellknippe and Li also proposed a tool called the Layout Bug Hunter (LBH) to detect three different types of layout anomalies [9]. LBH does not need any user specification. However, LBH relies on the underlying Fuse platform [11] to implement the GUI analytical work.

In this paper, we propose a layout anomaly detection tool called LAD (Layout Anomaly Detector) to perform visual testing on Android app layouts. LAD is designed to detect six types of layout anomalies: components missing, cropped text, component overlap, component overflow, component misalignment, and component/text scale maladaptation. To automate the detection process, a procedural script language is devised to specify the navigation procedure and the detection operations. LAD also supports two inspection modes: the standalone mode and the comparative mode. In the comparative mode, users specify a designated resolution to get gold standard layouts (GSLs) as the inspection templates. LAD automatically inspects all layouts under test (LUTs) in the testing resolutions against the GSLs. In the standalone mode, LAD works only for one testing resolution. To operate in a contiguous integration $(\mathrm{CI})$ testing process, LAD is designed as a native Java tool in a modular design.

The rest of this paper is organized as follows. Section 2 briefly reviews the related research work on the layout anomaly detection problem. Section 3 describes the layout anomalies detected in LAD and the LAD design. Section 4 presents the empirical study on LAD with four Android apps. Finally, Section 5 concludes this paper.

\section{RELATED WORK}

The layout anomaly detection problem is an important issue for traditional window-based interface design and Web application design [12-14]. For automatically detecting layout 
anomalies, the detection approaches can be mainly classified into two categories: the image comparing approach $[15,16]$ and the layout analysis approach [6-9].

The image comparing approach inspects the layout anomalies by making comparisons between two captured screenshots to find their pixel differences. Since there is no layout structure information in the contrasting process, this approach may suffer from the challenge to decide whether two images presented in difference screen sizes/resolutions are identical [9].

For the layout analysis approach, the layout information is used to detect the anomalies. In Galen Framework [6], users can program the test cases using Galen Specs Language to describe the relationships between two objects. However, Galen is mainly designed for Web apps. In ITArray Automotion Framework [7], a library is supported to perform visual testing on web and mobile pages. Users can describe the visual relationships using the library calls. As pointed in [9], however, these tools require users to provide detailed information about the placement of GUI objects and the validation parameters. If the GUI layouts are changed frequently, this scripting-in-detail approach may incur high maintenance costs.

In 2017, Shih et al. proposed a tool called UI-Explorer to automate the detection of layout anomalies [8]. UI-Explorer uses a GUI ripping engine to extract the layout information of all GUI components and automatically traverse all layouts under two different screen specifications. With UI-Explorer, users do not need to provide detailed visual information for testing. However, UI-Explorer considers only two types of layout anomalies: components missing and cropped text. Moreover, it provides only click events to traverse the layouts.

Hasellknippe and Li proposed a tool called the Layout Bug Hunter (LBH) for detecting three GUI layout anomalies: component overlap, component overflow, and component misalignment [9]. LBH employs a set of specified rules to detect layout anomalies. Therefore, $\mathrm{LBH}$ does not require any script for its visual testing. However, LBH does not consider the comparative approach. As discussed in [9], an intentional overlap design may be identified as an anomaly. Moreover, the current LBH design relies on the underlying Fuse platform [11].

\section{Design of LAyout ANOMALy Detection MECHANISMS}

This section first presents the layout anomalies considered in LAD. The design of the LAD architecture is described thereafter.

\section{A. Layout Anomalies}

In addition to the layout anomaly types discussed in the previous studies [8, 9], components missing, cropped text, component overlap, component overflow, and component misalignment, LAD considers one more type: component/text scale maladaptation. LAD supports two inspection modes for layout anomaly detection: the standalone mode and the comparative mode. In the standalone mode, LAD uses the predefined heuristic rules to decide the detection results. In the comparative mode, the layouts in a user-designated resolution are used as the inspection templates, which are the gold standard layouts (GSLs). The layouts under test (LUTs) in different screen sizes/resolutions will be investigated against the GSLs.

\section{1) Component Missing}

A component missing anomaly happens when some GUI component disappears in a different LUT screen size/resolution. Generally, this anomaly occurs when the screen size/resolution becomes smaller. As shown in Figure 1, the gender symbol at the bottom of the app in the $768 \times 1280$ GSL disappears in the $480 \times 800$ LUT.

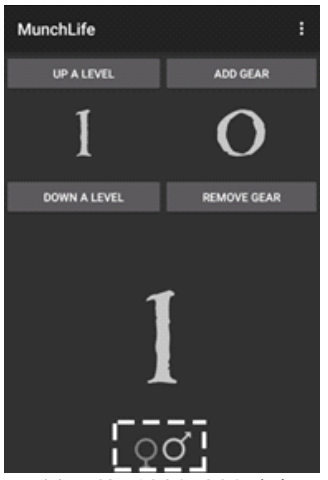

(a) $768 \times 1280,320 \mathrm{dpi}$

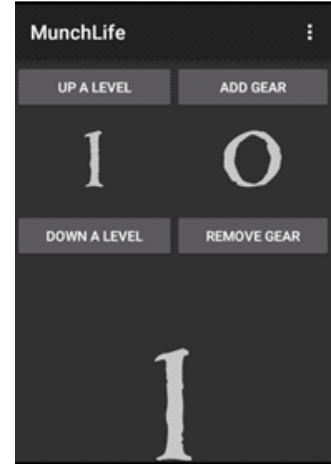

(b) $480 \times 800,240 \mathrm{dpi}$
Figure 1: Component missing anomaly

To detect component missing anomalies, LAD first retrieves the XML dumps of GSLs and LUTs. From the XML dumps, LAD calculates the total numbers of components of each GSL and the corresponding LUT. If these two numbers are not equal, the LUT is regarded as having a component missing anomaly.

\section{2) Cropped Text}

The cropped text anomaly happens when some text string correctly appears in the GSL, but it is cropped in the corresponding LUT. Generally, this anomaly occurs because the GUI components are squeezed in the LUT of a smaller screen resolution. As shown in Figure 2, when the $768 \times 1280$ GSL resolution is shrunk to the $480 \times 800$ LUT resolution, the text in the red box of Figure 2(b) is cropped. In this case, the app user cannot read the complete text.

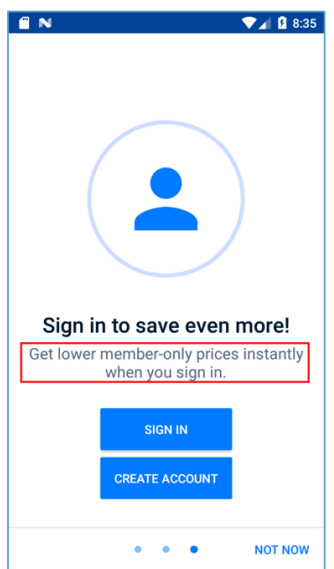

(a) $768 \times 1280,320 \mathrm{dpi}$

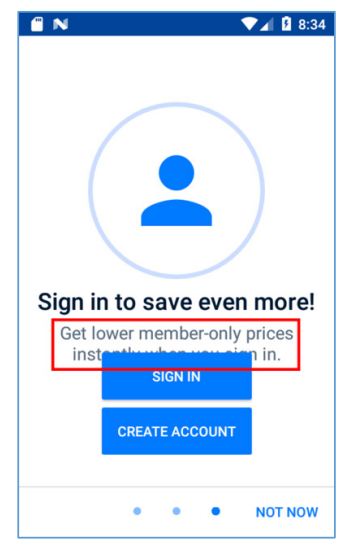

(b) $480 \times 800,240 \mathrm{dpi}$
Figure 2: Cropped text anomaly. 
LAD uses the same approach proposed in [8] to first obtain the coordinates of the text component $C_{1}$ in the GSL and invoke OpenCV (a computer vision library) to get a screenshot $S_{1}$ of $C_{1}$. LAD then invokes Tesseract (an OCR text recognizer) to perform the OCR work and get the OCR text $T_{1}$. Similarly, LAD gets the OCR text $T_{2}$ for the corresponding component $C_{2}$ in the LUT. For example, $T_{1}$ in the red box of Figure 2(a) is recognized as "Getlowermember-onlypricesinstantlywhenyousignin" and $T_{2}$ is recognized as "GetlowermemberonlypricesWMin-".

Because the OCR results usually have errors, LAD calculates the Levenshtein distance $\left(E d_{1}\right)$ between $T_{1}$ and the original string $C_{1}$.text. LAD also calculates $E d_{2}$ between $T_{2}$ and $C_{1}$.text. Therefore, if $\left|E d_{1}-E d_{2}\right| \geq T_{E D}$ where $T_{E D}$ is a predefined threshold, the LUT is regarded as having a cropped text anomaly.

\section{3) Component Overlap}

The component overlap anomaly happens when one GUI component covers a part of another GUI component. This may occur when a smaller screen size/resolution is used for LUTs. However, the original design may also contain this anomaly. Therefore, LAD detects it in both inspection modes.

Figure 3 shows an example in which Figure 3(a) is a correctly displayed GSL of a calculator app. In Figure 3(b), the dashed button area overlaps the background text area in the LUT. In this case, the app users cannot get the correct result.

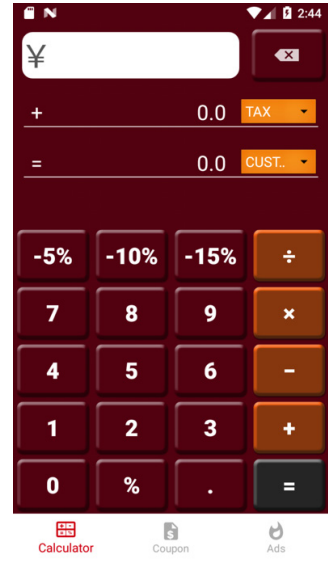

(a) $768 \times 1280,320 \mathrm{dpi}$

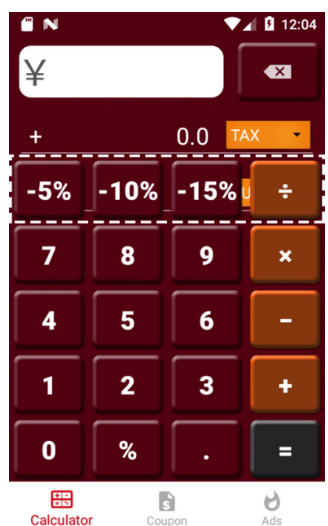

(b) $480 \times 800,240 \mathrm{dpi}$
Figure 3: Component overlap anomaly.

To detect component overlap anomalies, LAD retrieves the XML dumps of LUTs and obtains the coordinates of the upper left corner and the lower right corner of each component $C_{i}$ from the XML dumps. Then LAD calculates the areas of two components to see if these two areas are overlapped.

\section{4) Component Overflow}

When an app developer forgets to adjust the component size according to the new LUT resolution, a component overflow anomaly may occur in the LUT if the area of some component exceeds the screen boundary. In the worst case, users may not be able to correctly operate the component.

Figure 4 shows an example. In the $768 \times 1280 \mathrm{GSL}$ of Figure 4(a), both button components are completely displayed. In

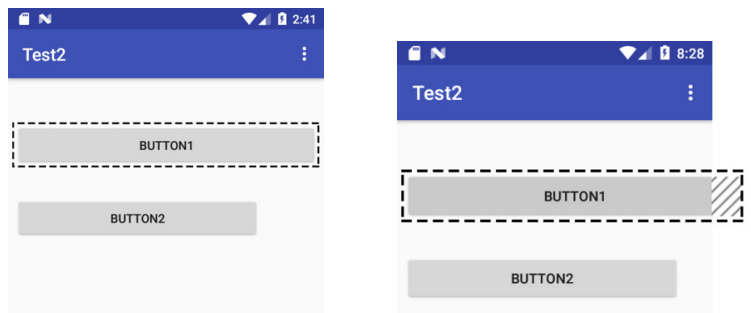

(a) $768 \times 1280,320 \mathrm{dpi}$

(b) $480 \times 800,240 \mathrm{dpi}$

Figure 4: Component overflow anomaly.

Figure 4(b), however, the area of BUTTON1 exceeds the boundary of the $480 \times 800$ LUT layout.

LAD also analyzes the XML dumps of LUTs to obtain the coordinate $\left(x_{i 1}, y_{i 1}\right)$ of the upper left corner of each component $C_{i}$ and its coordinate $\left(x_{i 2}, y_{i 2}\right)$ of the lower right corner. LAD also analyzes the component information to get the height $H_{i}$ and the width $W_{i}$. LAD compares these values with the screen height $S_{h}$ and the screen width $S_{w}$, respectively. If one of the following inequalities is satisfied, the LUT is regarded as having a component overflow anomaly.

- Horizontal boundary overflow

$$
x_{i 1}+W_{i}>S_{w}\left\|x_{i 2}<W_{i}\right\| x_{i 2}>S_{w}
$$

- Vertical boundary overflow

$$
y_{i 1}+H_{i}>S_{h}\left\|y_{i 2}<H_{i}\right\| y_{i 2}>S_{h}
$$

\section{5) Misalignment}

When a programmer designs a layout, the components are usually aligned in the layout. There are three kinds of vertical alignment: Align Top, Align Middle, and Align Bottom. Similarly, the horizontal alignment also has three kinds: Align Left, Align Center, and Align Right. When the component position is not properly adjusted with the resolution change, it is possible that a

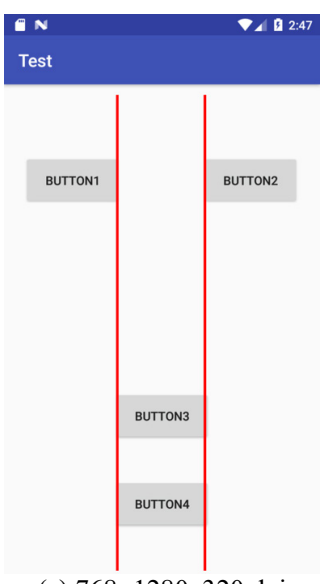

(a) $768 \times 1280,320 \mathrm{dpi}$

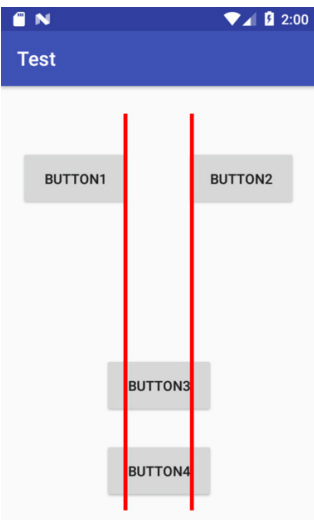

(b) $480 \times 800,240 \mathrm{dpi}$
Figure 5: Component misalignment anomaly. 
component misalignment anomaly occurs in the LUT resolution. For example, the $768 \times 1280$ GSL in Figure 5(a) illustrates that the left sides of BUTTON3 and BUTTON4 are aligned with the right side of BUTTON1 along with the red line. However, the $480 \times 800$ LUT in Figure 5(b) demonstrates that BUTTON1 is not horizontally aligned with BUTTON3 and BUTTON4.

To detect component misalignment anomalies, LAD makes comparisons for each pair of two components $C_{i}$ and $C_{j}$ using their coordinates and centers. However, a strict alignment judgement may incur many false alarms for two cases:

- Some very minor misalignments can be allowable because it is very hard for users to find them by eyes.

- $\quad$ Some misalignments are intentionally designed.

Therefore, LAD has two thresholds $D_{S}$ and $D_{L}$ to control the misalignment detection. $D_{S}$ is used to ignore the misalignments that are not easily recognized by users. In the current LAD design, the default value of $D_{S}$ is 2 pixels. $D_{L}$ is used to ignore the misalignments that are intentionally designed. In LAD, the default value of $D_{L}$ is 8 pixels. For example, if the left upper corner of $C_{i}$ is $\left(x_{i 1}, y_{i 1}\right)$ and the left upper corner of $C_{j}$ is $\left(x_{j 1}, y_{j 1}\right)$, the LUT is regarded as having a component misalignment anomaly when the following condition is satisfied.

$$
D_{S}<\left|x_{i 1}-x_{j 1}\right|<D_{L}
$$

\section{6) Component/Text Scale Maladaptation}

As the resolution of the screen changes, the sizes of the components and text characters may also change. If the size of a component is excessively reduced, users will have difficulty clicking the component or reading the text. The LUT is regarded as having a component/text scale maladaptation anomaly. LAD tackles this scale maladaptation problem separately for GUI components and text strings.

For GUI components, the size of an interactive component should be at least $44 \mathrm{pt} \times 44 \mathrm{pt}$ according to the Apple User Interface Design Guide [17]. In [18], however, Zea suggests that the minimum target size of the component should be $5 \mathrm{~mm} \times 5$ $\mathrm{mm}=30 \mathrm{px} \times 30 \mathrm{px}$. In the current LAD design, we use the size suggested by Zea as the threshold to determine the scale maladaptation anomaly for a component. If the size of a component is smaller than this threshold, a scale maladaptation

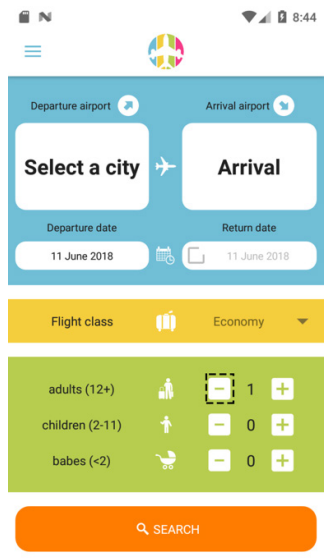

Figure 6: Component scale maladaptation anomaly in $768 \times 1280$ LUT.

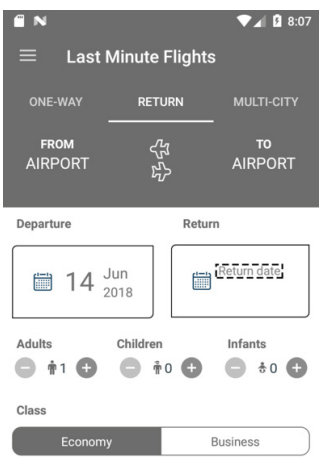

\section{SEARCH TICKEIS}

(a) Small text in $768 \times 1280$

\section{Return date $\prod_{t_{r}}^{\|_{r}}$}

(b) Detected text height $H_{T}=$ $0.35 \mathrm{~cm}$

Figure 7: Text scale maladaptation anomaly. anomaly occurs on the component. For example, Figure 6 illustrates that the dashed area is smaller than $5 \mathrm{~mm} \times 5 \mathrm{~mm}$ in the $768 \times 1280$ LUT.

For the text scale problem, Kahn and Lenk have pointed out that the font sizes of 12 and 14 points allow the user to read comfortably [19]. Tennant also points out that the 16-pixel font size is approximately equal to the 12-point font size which is suitable for reading [20]. Because the height of the 12-point font is approximately $0.42 \mathrm{~cm}$, LAD use this as a default threshold to determine if the scale of a text string is maladapted in the LUT.

Figure 7 illustrates an example in which LAD first gets the coordinates of the dashed box containing a string "Return date" and then invokes OpenCV to get its screenshot. LAD then uses a text edge detection algorithm [21] to obtain the height $H_{T}$ of the string as shown in Figure $7(\mathrm{~b})$. If $H_{T}<0.42 \mathrm{~cm}$, the LUT is regarded as having a text scale maladaptation anomaly.

\section{B. LAD Architecture}

\section{1) Script Language}

To automate the visual testing, a script language is devised in LAD for controlling the testing procedure. With the test scripts, LAD can automatically enter text inputs into the

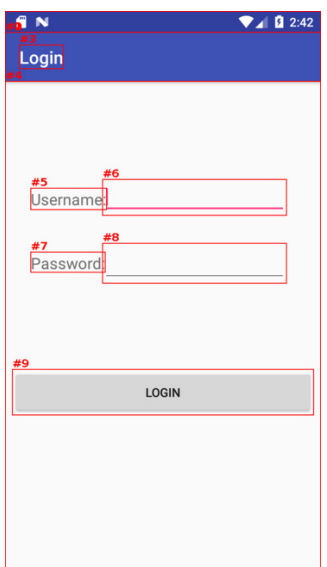

(a) Component marking

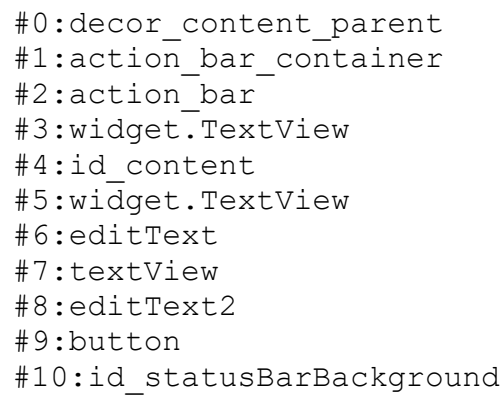

(b) Component list
Figure 8: Component marks and the list of their IDs. 


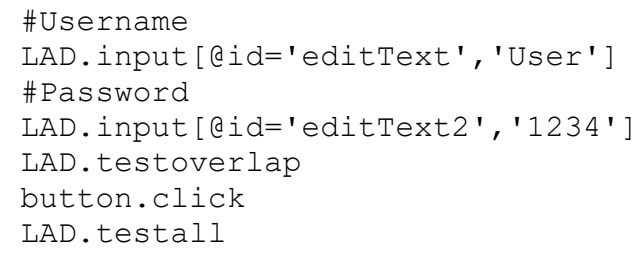

Figure 9: An example script.

components and navigate to different layouts. In order to let the script writer know the component IDs of each layout, LAD has a companion tool LADMark to extract the component IDs and mark each component on the layout screenshot as shown in Figure 8(a). Figure 8(b) is the component list.

Figure 9 shows a script example based on the list in Figure 8(b). A starting "\#" is used to denote a comment. On the second line, "LAD. input [ [id='editText', 'User']" is used to enter the text input "User" for the username. On line 4, "LAD. input [@id='editText2', '1234']" is used to input the password "1234". On line 5, LAD. testoverlap instructs LAD to perform anomaly detection for component overlap. On line 6, button. click tells LAD to click the LOGIN button such that the app moves to the next layout. Finally, LAD. testall instructs LAD to perform anomaly detection for all kinds of anomalies.

\section{2) Architecture Design}

Figure 10 shows the architecture of the LAD. The system reads the AUT, the LUT screen resolution, the GSL screen resolution, and the testing scripts. Then, LAD analyzes the testing scripts and automatically navigates the GSLs and LUTs for testing.

Finally, LAD collects the testing results and generates the detection report. To help the developer find the corresponding places of the detected anomalies, the report contains the

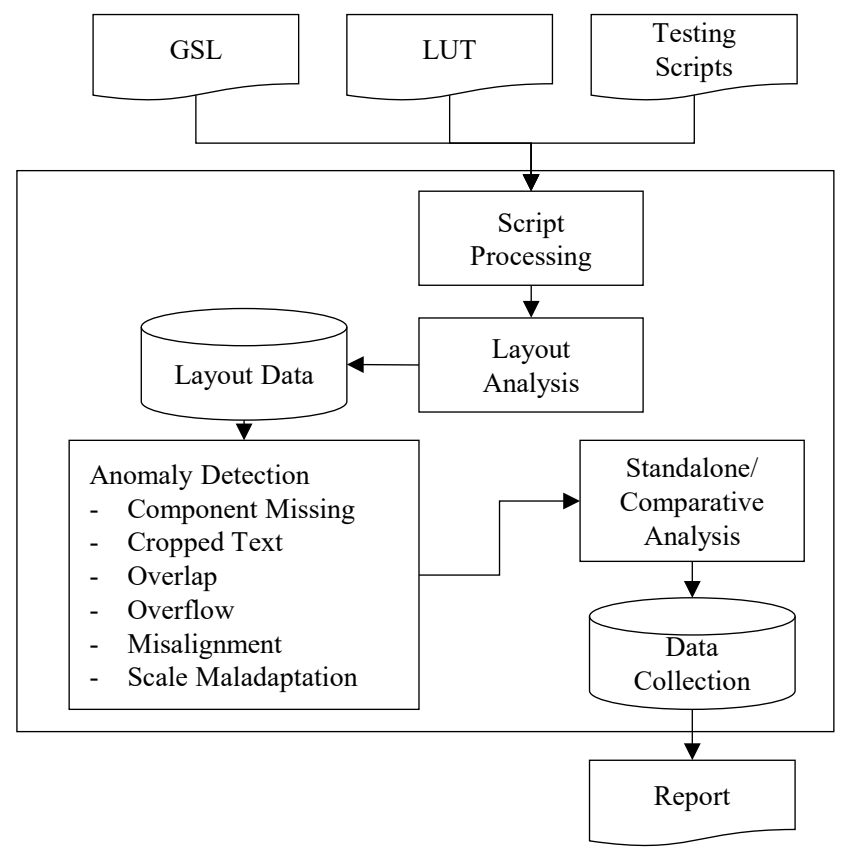

Figure 10: Detection system architecture.

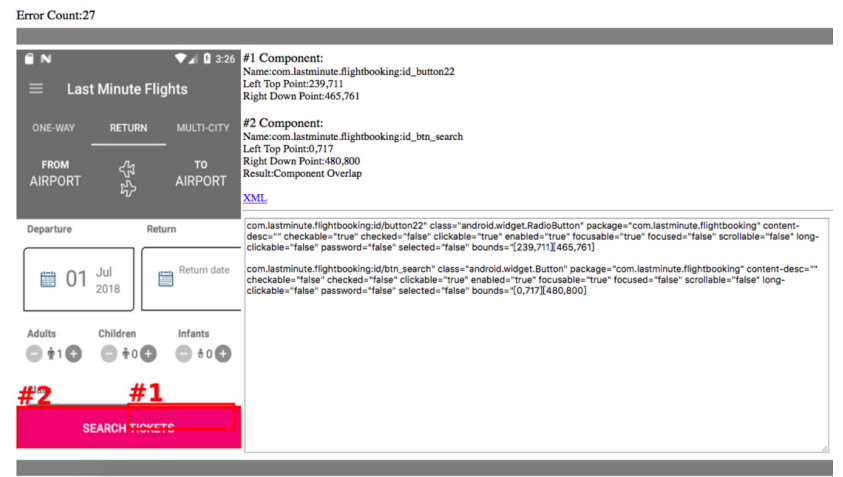

Figure 11: A detection report example.

screenshots, the detection results, and the XML information of the problematic components. Figure 11 illustrates a report example.

\section{EXPERIMENTS}

To evaluate the effectiveness of the proposed anomaly detection mechanism, we have conducted experiments with four Android apps. This section describes the experimental results.

\section{A. Experimental Environment}

We have implemented a prototype for experiments. The LAD prototype was executed on a PC running with 16 GB RAM and an Intel i5-6500 3.20 GHz CPU. The operating system was Ubuntu 16.04 64bit. The Android emulator was the built-in emulator of Android Studio 3.1 emulating Google 7.0.0 API 24. The resolutions of GSL and LUT were $768 \times 1280$ and $480 \times 800$, respectively. Four investigated apps are as follows:

- MunchLife 1.4.4 (ML): This app is used to track the character level for a card game Munchkin.

- $\quad$ Last Minute Flights Booking 2.0 (LMFB): This app allows users to compare flight and hotel information provided by airlines and travel agencies. Users can use it to find the most suitable flights and hotels.

- Priceline 4.34.170 (PL): This app allows users to quickly plan their travel itineraries, including booking cheap flights, hotels and rental cars.

- MyAir365 1.06 (MA): This app allow users to easily order low-cost airlines.

The main reason for choosing these four apps is that they all have layout anomalies. Therefore, they can be used to demonstrate the detection performance. In order to clearly present the detection results, this paper shows the experimental results using only the first page of each app.

\section{B. Experimental Results}

As shown in Table 1, the numbers are the anomalies detected in the experiments. For anomalies of components missing, cropped text, component overlap, component overflow, and component misalignment, the results are obtained in the comparative mode. For scale maladaptation anomalies, the results are measured in the standalone mode with the $768 \times 1280$ 
TABLE I. DETECTION RESULTS OF LAD.

\begin{tabular}{|l|c|c|c|c|}
\hline & ML & LMFB & PL & MA \\
\hline Comp. missing & 1 & 0 & 0 & 0 \\
\hline Cropped text & 0 & 3 & 4 & 0 \\
\hline Overlap & 2 & 10 & 0 & 7 \\
\hline Overflow & 0 & 0 & 0 & 0 \\
\hline Misalignment & 0 & 6 & 0 & 11 \\
\hline Comp. scale maladapt. & 0 & 5 & 8 & 8 \\
\hline Text scale maladapt. & 5 & 10 & 8 & 3 \\
\hline
\end{tabular}

resolution. The manual inspections show that these detection results can effectively discover many anomalies which may be ignored by inspectors.

In the experiments, some false alarms were found in the standalone mode because the default heuristic thresholds are not appropriate for some apps and scree specifications. However, these false alarms can be eliminated in the comparative mode because the GSLs can filter out their detections in the LUTs.

\section{CONCLUSION}

Since Android has the severe fragmentation problem, visual testing has received considerable attentions in recent research. Traditionally, developers need to spend much time in manually checking layouts with different screen resolutions. This manual inspection process is time-consuming and error-prone.

In this paper, we present the design of an anomaly detection tool LAD which can be used to detect six types of layout anomalies. To automatically detect these anomalies in different layouts, a procedural script language is devised to specify the app navigation procedure and the detection operations. To operate in a contiguous integration (CI) testing environment, LAD is designed as a native Java tool in a modular design. Moreover, LAD supports two inspection modes: the standalone mode and the comparative mode. The comparative mode facilitates the avoidance of false alarms in the detection results.

In our future work, we plan to conduct comprehensive investigations with more apps to study the effectiveness of LAD. Human inspectors will also involve the experiments to make performance comparisons by measuring more metrics, such as accuracy, precision, and recall. Moreover, the functionality of LAD will be enhanced. The current design does not have a plugin interface. Therefore, it is inflexible for users who want to add a new module for a new anomaly. The algorithms for anomaly detection will be also improved further and the practical applicability of the detection reports will be enhanced to help developers quickly detect the layout anomalies and improve the quality of Android apps.

\section{ACKNOWLEDGEMENT}

This work was supported in part by Ministry of Science and Technology, Taiwan under grant MOST 107-2221-E-155-012. The authors would also like to express many thanks to the anonymous reviewers for their precious suggestions.

\section{REFERENCES}

[1] AppBrain, "Android apps on Google Play", https://www.appbrain.com/stats/number-of-android-apps, last accessed on March 1, 2019.
[2] D. Amalfitano, A. R. Fasolino, P. Tramontana, S. De Carmine, and A. M. Memon, "Using GUI Ripping for Automated Testing of Android Applications", in Proceedings of the 27th IEEE/ACM International Conference on Automated Software Engineering (ASE 2012), pp. 258261, 2012.

[3] D. Amalfitano, A. R. Fasolino, P. Tramontana, B. D. Ta, and A. M. Memon, "MobiGUITAR: Automated Model-Based Testing of Mobile Apps", IEEE Software, vol. 32, no. 5, pp. 53-59, 2015.

[4] C. Hu, and I. Neamtiu, "Automating GUI Testing for Android Applications", in Proceedings of the 6th International Workshop on Automation of Software Test (AST '11), pp. 77-83, 2011.

[5] H.-L. Wen, C.-H. Lin, T.-H. Hsieh, and C.-Z. Yang, "PATS: A Parallel GUI Testing Framework for Android Applications", in Proceedings of the 2015 IEEE 39th Annual Computer Software and Applications Conference (COMPSAC 2015), pp. 210-215, 2015.

[6] Galen Framework, "Galen Framework", 2019, http://galenframework.com/, last accessed on March 1, 2019.

[7] D. Zaiats, "ITArray Automotion Framework", 2019, https://automotion.itarray.net/, last accessed on March 1, 2019.

[8] Y.-A. Shih, Y.-P. Chang, and C.-Z. Yang, "An Automated Detection Framework for Testing Visual GUI Layouts of Android Applications", in Proceedings of the 7th International Workshop on Computer Science and Engineering (WCSE 2017), pp. 544-548, 2017.

[9] K. F. Hasselknippe and J. Li, "A Novel Tool for Automatic GUI Layout Testing", in Proceedings of the 24th Asia-Pacific Software Engineering Conference (APSEC 2017), pp. 695-700, 2017.

[10] OpenSignal, "Android Fragmentation Visualized (August 2014)" https://opensignal.com/reports/2014/android-fragmentation/, 2014, last accessed on December 20, 2017.

[11] Fusetools AS, "Fusetools - We Make Apps Easy," https://www.fusetools.com, 2019, last accessed on March 1, 2019.

[12] E. Ch'ng, and D. C. L. Ngo, "Screen Design: A Dynamic Symmetry Grid Based Approach”, Displays, vol. 24, pp. 125-135, 2003.

[13] H. Song, H. Liu, and D. Chen, "An Automatic GUI Adjustment Method for Mobile Computing", in Proceedings of the 2011 IEEE International Conference on Computer Science and Automation Engineering (CSAE 2011), pp. 206-210, 2011.

[14] S. Hallé, N. Bergeron, F. Guerin, G. L. Breton, and O. Beroual, "Declarative Layout Constraints for Testing Web Applications", Journal of Logical and Algebraic Methods in Programming, vol. 85, no. 5, pp. 737-758, 2016

[15] Applitools, “Applitools Eyes", https://applitools.com/, 2019, last accessed on March 1, 2019.

[16] J. Cryer, "PhantomCSS", https://github.com/HuddleEng/PhantomCSS, 2019, last accessed on March 1, 2019.

[17] Apple Inc., "Human Interface Guidelines - Adaptivity and Layout," https://developer.apple.com/design/human-interfaceguidelines/ios/visual-design/adaptivity-and-layout/, 2019, last accessed on March 1, 2019.

[18] R. Zea, "Mastering Responsive Web Design with HTML5 and CSS3", Packt Publishing Ltd., 2015.

[19] P. Kahn, and K. Lenk, "Design: Principles of Typography for User Interface Design”, Interactions, vol. 5, no. 6, pp. 15-29, 1998.

[20] D. B. Tennant, "16 Pixels Font Size: For Body Copy. Anything Less Is A Costly Mistake", $\quad$ Smashing, 2011. https://www.smashingmagazine.com/2011/10/16-pixels-body-copyanything-less-costly-mistake/

[21] C. Liu, C. Wang, and R. Dai, "Text Detection in Images Based on Unsupervised Classification of Edge-based Features", in Proceedings of the 2005 8th International Conference on Document Analysis and Recognition (ICDAR '05), pp. 610-614, 2005. 